\title{
PARAMETRIC AMPLIFICATION IN MAGNETIZED NONDEGENERATE SEMICONDUCTING PLASMAS*
}

\author{
K.L. JAT ${ }^{\dagger}$, A. NEOGI AND S.GHOSH \\ School of Studies in Physics, Vikram University, Ujjain 456010, India \\ (Received October 23, 1989; in final version September 10, 1990)
}

\begin{abstract}
Using the straightforward coupled-mode theory, the parametric amplification is analytically investigated in magnetized piezoelectric as well as non-piezoelectric semiconductors. The origin of nonlinear interaction is taken to be in the second-order optical susceptibility $\chi^{(2)}$ arising from the nonlinear induced current density. The threshold value of the pump electric field $E_{0 \mathrm{th}}$ is obtained for crystals. $E_{0 \text { th }}$ is found to decrease with the rise in magnetic field and fall with the rise in scattering angle. Parametric gain constants are obtained for different situations of practical interest, i.e. (i) for piezoelectric coupling only $g_{\mathrm{p}}$, (ii) for deformation potential coupling only $g_{\mathrm{d}}$, (iii) for both the couplings $g_{\mathrm{b}}$. Numerical investigations reveal that the magnetic field increases the gain. Maximum gains are obtained for backward scattered mode. $g_{\mathrm{d}}$ is always found to be less than $g_{\mathrm{p}}$ and $g_{\mathrm{b}}$. It is also found that $g_{\mathrm{p}}$ and $g_{\mathrm{b}}$ both are identical up to $k \approx 3 \times 10^{7} \mathrm{~m}^{-1}$ and for $k \geq 3 \times 10^{7} \mathrm{~m}^{-1}$, $g_{\mathrm{b}}$ exceeds $g_{\mathrm{p}}$.
\end{abstract}

PACS numbers: 42.65.Ky, 72.20.Ht, 73.50.Rb, 78.20.Hp

\section{Introduction}

Optical nonlinearity is manifested by changes in the optical parameters of a medium as the intensity of the impressed light wave is increased or when one or more light waves are introduced. The phenomenon of parametric interaction of coupled waves exhibit a vital role in nonlinear optics. The motivation which led to a rapid development in the field of nonlinear optics is the possibility of exploiting

"Work done under the D.S.T. research project "Plasma Effects in Semiconductors: Wa ves and Instabilities".

tPermament address: Department of Physics, Government College, Neemuch, (M.P.) India. 
the nonlinear behaviour in various solid state devices such as frequency converters, parametric amplifiers and oscillators, optical phase conjugators etc. [1-3]. It is a well-known fact that the origin of parametric interaction lies in the second-order nonlinear optical susceptibility $\chi^{(2)}$ of the medium. Earlier Flytzanis [4] has reviewed the progress made in the study of $\chi^{(2)}$ in different frequency regimes in solids as well as in diluted media. $\chi^{(2)}$, the lowest order susceptibility, is a third rank tensor which is, nonvanishing in a medium which lacks inversion symmetry. The polarization which is quadratic in nature in terms of field amplitude leads to the optical phenomena of second harmonic generation (SHG), sum and difference frequency generation etc. In general, the terms in $\chi^{(2)}$ provide a coupling between the set of three electromagnetic waves; each of which is characterised by frequency $\omega_{i}$, wave number $k_{i}$, state of polarization $\varepsilon_{i}$, as well as complex amplitude $E_{i}=A_{i} \exp \left(\mathrm{i} \omega_{i} t\right)$. Attempts were made to explain the experimentally observed behaviour of $\chi^{(2)}$ on the basis of multiple valence and conduction bands, but the agreement was not very encouraging [5,6]. All these studies [4-6] were mainly covered with the induced polarizations arising from bound-electron nonlinearities. The sum rules for nonlinear susceptibilities in solids have been studied by Peiponen [7].

It is observed that the crystalline nonlinear media offer the greatest device potential. This is because $\chi^{(2)}$ is nonzero for noncentrosymmetric (NCS) crystals and secondly the birefringence of a crystalline medium could be used to phase match velocities of fundamental and harmonic radiations by compensating the material dispersion. However, for nonlinear optical applications, nonlinear crystals, should satisfy four basic criteria, namely adequate nonlinearity, optical transparency, proper birefringence for phase matching and sufficient resistance to optical damage by intense optical irradiation. The doped semiconductors are found to be transparent to photons of energy below their energy band gap and thereby prove to be advantageous hosts. The properties of nonlinear materials are better understood when discussed with reference to nonlinear devices and the theory of nonlinear interactions.

The parametric interaction of acoustic waves with microwave electric field in piezoelectric semiconductors was discovered by Economou and Spector [8]. The importance of the effect of a d.c. magnetic field on parametric action was very well emphasized by Cohen [9]. The parametric excitation of hybrid mode has been studied by Ghosh and Agarwal [10]. Recently Aghamkar et al. [11] have reported the parametric dispersive as well as absorptive characteristics while calculating $\chi^{(2)}$ originating from the finite induced current density produced in a semiconducting medium. Motivated by the intense interest in the field of study of parametric interaction based on $\chi^{(2)}$, in the present paper the authors have made an attempt to investigate parametric amplification process originating from $\chi^{(2)}$, in a nondegenerate $n$-InSb crystal of NCS nature when a magnetostatic field is applied perpendicular to the direction of the pump wave propagation. Since Aghamkar et al. [11] have concluded that the highly doped semiconductors are best suited for observing parametric interactions, the authors in the present paper have confined themselves to the highly doped crystals only. 


\section{Theoretical formulations}

We consider the hydrodynamic model of a homogeneous, nondegenerate $n$-type semiconductor plasma having both piezoelectric as well as deformation potential couplings and the medium is of infinite extent with electrons as carriers. This model restricts the validity of the analysis to the limit $k l \ll 1$, where $k$ is the wave number and $l$ is the mean free path of the electrons. In order to study parametric interaction processes originating from the effective nonlinear optical susceptibility $\left(\chi_{\mathrm{EN}}\right)$ the medium is subjected to the magnetic field $\boldsymbol{B}_{0}$ (along $\boldsymbol{z}$-axis) perpendicular to the propagation direction ( $x$-axis) of spatially uniform high frequency pump electric field $\boldsymbol{E}_{0} \exp \left(-\mathrm{i} \omega_{0} t\right)$. The scattered waves are propagating along a direction making an arbitrary angle $\theta$ with the pump wave propagation direction, i.e. propagating in $x-z$ plane making an angle $\theta$ with $x$-axis. Thus $\theta$ is the scattering angle, i.e. the angle between $k_{0}$ and $k_{1}$. We apply the coupled mode theory [12] to obtain a simplified expression for the acoustic waves via density perturbation.

The basic equations used are as follows:

$$
\begin{gathered}
\frac{\partial v_{0}}{\partial t}+\nu v_{0}=-\frac{e}{m}\left[E_{0}+v_{0} \times B_{0}\right] \\
\frac{\partial v_{1}}{\partial t}+\nu v_{1}+\left(v_{0} \frac{\partial}{\partial x}\right) v_{1}=-\frac{e}{m}\left[E_{1}+v_{1} \times B_{0}\right] \\
v_{0} \frac{\partial n_{1}}{\partial x}+n_{0} \frac{\partial v_{1}}{\partial x}=-\frac{\partial n_{1}}{\partial t} \\
\frac{\partial E_{\mathrm{s}}}{\partial x}+\frac{\beta}{\varepsilon} \frac{\partial^{2} u}{\partial x^{2}}-\frac{C_{\mathrm{d}}}{e} \frac{\partial^{3} u}{\partial x^{3}}=-\frac{n_{1} e}{\varepsilon} \\
\rho \frac{\partial^{2} u}{\partial t^{2}}+2 \gamma_{\mathrm{s}} \rho \frac{\partial u}{\partial t}+\beta \frac{\partial E_{\mathrm{s}}}{\partial x}+\frac{C_{\mathrm{d}} \varepsilon}{e} \frac{\partial^{2} E_{\mathrm{s}}}{\partial x^{2}}=C \frac{\partial^{2} u}{\partial x^{2}}
\end{gathered}
$$

Equations (1) and (2) represent the zeroth- and first-order momentum transfer equations, respectively, in which $v_{0}$ and $v_{1}$ are the zeroth- and first-order oscillatory fluid velocities having effective mass $m$ and charge $-e$ and $\nu$ is the phenomenological electron collision frequency. Equation (3) represents the continuity equation for electrons, where $n_{0}$ and $n_{1}$ are the equilibrium and perturbed electron densities, respectively. The Poisson equation (4) gives the space charge field $E_{\mathrm{s}}$ in which the second and the third terms on the left hand side give the piezoelectric and deformation potential contribution to polarization, respectively. $\varepsilon, \beta$ and $C_{\mathrm{d}}$ are the scalar dielectric, piezoelectric and deformation potential constants of the semiconductor, respectively. Equation (5) describes the motion of the lattice in a crystal having piezoelectric and deformation potential couplings both. In this equation $\rho, u, \gamma_{\mathrm{s}}$ and $C$ being the mass density of the crystal, displacement of the lattice, phenomenological damping parameter of acoustic mode and crystal elastic constant, respectively. In (2) we have neglected the effect due to $v_{0} \times B_{1}$ by assuming that the shear acoustic wave is propagating along such a direction of the crystal that it produces a longitudinal electric field, e.g. in $n-\operatorname{InSb}$, if $k$ is 
taken along (011) and the lattice displacement $u$ is along (100) the electric field induced by the wave is a longitudinal field [13].

In a highly doped semiconductor the low frequency acoustic wave $\left(\omega_{s}\right)$ as well as the pump electromagnetic wave $\left(\omega_{0}\right)$ produce density perturbations $\left(n_{1}\right)$ at the respective frequencies in the medium which can be obtained by using the standard approach [14]. Considering the low frequency perturbations $\left(n_{\mathrm{s}}\right)$ to be proportional to exp $\left[\mathrm{i}\left(k_{\mathrm{s}} x-\omega_{\mathrm{s}} t\right)\right]$, while $v_{0}$ varies as $\exp \left(-\mathrm{i} \omega_{0} t\right)$ and neglecting the Doppler shift under the assumption that $\omega_{0} \gg \nu>k v_{0}$, we get from Eqs. (1-4) as follows:

$$
\frac{\partial^{2} n_{1}}{\partial t^{2}}+\nu \frac{\partial n_{1}}{\partial t}+n_{1} \bar{\omega}_{\mathrm{p}}^{2}+\frac{n_{0} e \beta}{m \varepsilon} \frac{\partial^{2} u}{\partial x^{2}}-\frac{n_{0} C_{\mathrm{d}}}{m} \frac{\partial^{3} u}{\partial x^{3}}=-\bar{E} \frac{\partial n_{1}}{\partial x}
$$

where $\bar{E}=-\left[(e / m) E_{0}+\omega_{\mathrm{c}} v_{0 y}\right]$, and $\bar{\omega}_{\mathrm{p}}^{2}=\omega_{\mathrm{p}}^{2} \nu^{2} /\left(\nu^{2}+\omega_{\mathrm{c}}^{2}\right)$, in which $\omega_{\mathrm{p}}^{2}=$ $n_{0} e^{2} /(m \varepsilon)$ is the electron plasma, $\nu$ being the electron collision and $\omega_{\mathrm{c}}=\left(e B_{0} / m\right)$ being the cyclotron frequencies, respectively.

The density perturbations associated with the phonon mode (viz. $n_{s}$ ) and the scattered electromagnetic waves $\left(n_{\mathfrak{f}}\right)$ arising due to the three wave parametric interaction will propagate at the generated frequencies $\omega_{\mathrm{s}}$ and $\omega_{0} \pm \omega_{\mathrm{s}}$ respectively. For these modes the phase matching condition $\omega_{0}=\omega_{1}+\omega_{\mathrm{s}}$ and $k_{0}=k_{1}+k_{\mathrm{s}}$, i.e. the energy and momentum conservation relations should be satisfied. Now since $\theta$ is the angle between $k_{1}$ and $k_{0}$, thus in writing the conservation equations we have assumed $k_{1 y}=0$, i.e. the scattered wave to propagate in the $x-z$ plane. It must be mentioned here that these conservation equations could be satisfied over a wide range of scattering angle. Now for spatially uniform laser irradiation $\left|k_{0}\right| \approx 0$ and one obtains $\left|k_{1}\right|=\left|k_{\mathrm{s}}\right|=|\boldsymbol{k}|$ (say). On resolving equation (6) into two components (fast and slow) by denoting $v=v_{\mathrm{f}}+v_{\mathrm{s}}$ and $n=n_{\mathrm{f}}+n_{\mathrm{s}}$ under rotating wave approximation (RWA) one obtains:

$$
\frac{\partial^{2} n_{\mathrm{f}}}{\partial t^{2}}+\nu \frac{\partial n_{\mathrm{f}}}{\partial t}+\bar{\omega}_{\mathrm{p}}^{2} n_{\mathrm{f}}=-\bar{E} \frac{\partial n_{\mathrm{s}}^{*}}{\partial x}
$$

and

$$
\frac{\partial^{2} n_{\mathrm{s}}}{\partial t^{2}}+\nu \frac{\partial n_{\mathrm{s}}}{\partial t}+\bar{\omega}_{\mathrm{p}}^{2} n_{\mathrm{s}}+\frac{n_{0} e \beta}{m \varepsilon} \frac{\partial^{2} u}{\partial x^{2}}-\frac{\dot{n}_{0} C_{\mathrm{d}}}{m} \frac{\partial^{3} u}{\partial x^{3}}=-\bar{E} \frac{\partial n_{\mathrm{f}}^{*}}{\partial x} .
$$

In the above analysis we have restricted ourselves only to the Stokes component $\left(\omega_{0}-\omega_{s}\right)$ of the scattered electromagnetic waves. One can easily infer from (7) that the slow and fast components of the density perturbations are coupled to each other via the pump electric field. Thus the presence of the pump electric field is the fundamental necessity for the parametric interactions to occur. From Eqs. (5), (7a) and (7b) one obtains the expression for $n_{\mathrm{s}}$ as:

$$
\begin{array}{r}
n_{\mathrm{s}}=\frac{\mathrm{ien} n_{0} k_{x}^{3} E_{\mathrm{s}}\left(\beta^{2}+C_{\mathrm{d}}^{2} \varepsilon^{2} k_{x}^{2} / e^{2}\right)}{m \rho \varepsilon\left(\omega_{\mathrm{s}}^{2}-k_{x}^{2} v_{\mathrm{s}}^{2}+2 \mathrm{i} \gamma_{\mathrm{s}} \omega_{\mathrm{s}}\right)} \\
\times\left[\left(\bar{\omega}_{\mathrm{p}}^{2}-\omega_{\mathrm{s}}^{2}\right)-\mathrm{i} \omega_{\mathrm{s}} \nu-\frac{k_{x}^{2}|\bar{E}|^{2}}{\left(\dot{\omega}_{\mathrm{p}}^{2}-\omega_{1}^{2}+\mathrm{i} \omega_{1} \nu\right)}\right]^{-1}
\end{array}
$$


where $k_{\mathrm{x}}=k \cos \theta$ and $v_{\mathrm{s}}^{2}=(C / \rho) ; v_{\mathrm{s}}$ being the velocity of the acoustic wave. In the present report in order to study the effect of nonlinear current density on the induced polarization in a magnetized highly doped semiconductor, the effect of the transition dipole moment is neglected while analysing parametric interaction in the crystal. It is evident from the above expression that $n_{\mathbf{s}}$ depends upon the various powers of pump intensity, $I=\frac{1}{2} \eta \varepsilon_{0} c_{0}\left|E_{0}\right|^{2} ; \eta$ and $c_{0}$ being the background refractive index of the crystal and the velocity of light in vacuum, respectively. This produced density perturbation thus affecting the propagation characteristics . of the scattered waves, which can be studied by employing the electromagnetic wave equation:

$$
\nabla \times \nabla \times \boldsymbol{E}_{1}=\frac{-1}{C_{\mathrm{L}}^{2}} \frac{\partial^{2} E_{1}}{\partial t^{2}}-\mu_{0} \frac{\partial J_{1}}{\partial t}
$$

where $C_{\mathrm{L}}=\left(\mu_{0} \varepsilon_{0} \varepsilon_{\mathrm{L}}\right)^{-1 / 2}$ is the velocity of light in the medium and $J_{1}$ is the perturbed current density and $\varepsilon_{\mathrm{L}}=\varepsilon / \varepsilon_{0}$.

The Stokes component of the induced current density is given by

$$
J_{1}=-n_{\mathrm{s}}^{*} e v_{0}
$$

Using Eqs. (8) and (10a) one gets:

$$
J_{1}=\frac{-\mathrm{i} e k_{x}^{3} v_{\mathrm{s}}^{2} \varepsilon \omega_{\mathrm{p}}^{2} \omega_{0} E_{\mathrm{s}}^{*} E_{0}\left(\mathcal{K}^{2}+L^{2} k_{x}^{2}\right)}{2 m \gamma_{\mathrm{s}} \omega_{\mathrm{s}}\left(\omega_{\mathrm{c}}^{2}-\omega_{0}^{2}\right)}\left[{\delta^{\prime}}^{2}+\mathrm{i} \omega_{\mathrm{s}} \nu-\frac{k_{x}^{2}|\bar{E}|^{2}}{\left(\delta^{2}-\mathrm{i} \omega_{1} \nu\right)}\right]^{-1}
$$

where $\mathcal{K}^{2}=\beta^{2} / \varepsilon C, L^{2}=\left(C_{d} / e\right)^{2}(\varepsilon / C), \delta^{\prime 2}=\bar{\omega}_{\mathrm{p}}^{2}-\omega_{0}^{2}, \delta^{2}=\bar{\omega}_{\mathrm{p}}^{2}-\omega_{1}^{2}$ and $\omega_{1}=\omega_{0}-\omega_{\mathrm{s}}$. In deriving Eqs. (10) we have used the expression for the components of $v_{0}$ (along $\mathrm{x}$ - and $\mathrm{y}$-directions) which is the oscillatory electron fluid velocity in the presence of the pump and the magnetostatic fields. Using Eq. (1), these expressions are obtained as:

$$
v_{0 x}=\frac{\bar{E}}{\left(\nu-\mathrm{i} \omega_{0}\right)} \text { and } v_{0 y}=-(e / m) \frac{\omega_{\mathrm{c}} E_{0}}{\left(\omega_{\mathrm{c}}^{2}-\omega_{0}^{2}\right)}
$$

Henceforth treating the induced polarization $\boldsymbol{P}_{1}$ as the time integral of the current density $J_{1}$, one may write:

$$
P_{1}=\int J_{1} \mathrm{~d} t
$$

Now the second order susceptibility in the coupled mode scheme obtained by using equations (10) to (12), on neglecting the induced polarization due to transition dipoles, comes out to be as follows:

$$
\chi_{\mathrm{EN}}^{(2)}=\frac{e k_{x}^{3} v_{\mathrm{s}}^{2} \varepsilon_{\mathrm{L}} \omega_{\mathrm{p}}^{2} \omega_{0}\left(\mathcal{K}^{2}+L^{2} k_{x}^{2}\right)}{2 m \gamma_{\mathrm{s}} \omega_{\mathrm{s}}\left(\omega_{\mathrm{c}}^{2}-\omega_{0}^{2}\right) \omega_{1}}\left[\delta^{\prime 2}+\mathrm{i} \omega_{\mathrm{s}} \nu-\frac{k_{x}^{2}|\bar{E}|^{2}}{\left(\delta^{2}-\mathrm{i} \omega_{1} \nu\right)}\right]^{-1} .
$$

As susceptibility is a complex quantity, it can be expressed as:

$$
\chi_{\mathrm{EN}}^{(2)}=\chi_{\mathrm{EN}_{\mathrm{r}}}^{(2)}+\mathrm{i} \chi_{\mathrm{EN}_{\mathrm{i}}}^{(2)}
$$


From Eqs. (13) and (14) one may infer that the total crystal susceptibility is influenced by the free carrier concentration through plasma frequency $\omega_{\mathrm{p}} \neq 0$ and by magnetostatic field through cyclotron frequency $\omega_{c} \neq 0$. The dispersion characteristics of the scattered wave in a parametric process from $\left(\chi_{\mathrm{EN}_{\mathrm{r}}}^{(2)}\right)$ and the parametric gain through $\chi_{\mathrm{EN}_{\mathrm{i}}}^{(2)}$ can be seen from Eq. (13).

Here in the present paper we will confine ourselves to the study of parametric gain in the presence of a magnetostatic field and deformation potential coupling.

The parametric amplification in a doped semiconductor is given by:

$$
\alpha_{\mathrm{EN}}=\left(\frac{\omega_{1}}{\eta c_{0}}\right) \chi_{\mathrm{EN}}^{(2)}
$$

where $\alpha_{\mathrm{EN}}$ is the effective nonlinear absorption coefficient. The nonlinear growth of the signal $\omega_{1}=\omega_{0}-\omega_{\mathrm{s}}$ as well as idler $\left(\omega_{\mathrm{s}}\right)$ is possible only if $\alpha_{\mathrm{EN}}$ obtained from Eq. (15) is negative.

\section{Parametric growth}

Restricting ourselves to the analytical investigations and numerical estimations of the parametric growth in isotropic and magnetostatic semiconductors in heavily doped regime, i.e. $\bar{\omega}_{\mathrm{p}} \approx \omega_{0}\left(\approx \omega_{1}\right)$ with $\bar{\omega}_{\mathrm{p}} \gg \nu\left(\approx \omega_{\mathrm{s}}\right)$, Eq. (13) can be solved for complex $\chi \mathrm{EN}$ to get:

$$
\chi_{\mathrm{EN}_{x}}^{(2)}=\frac{e \varepsilon_{\mathrm{L}} A k_{x} \omega_{\mathrm{p}}^{2} \omega_{0}\left(1+L^{2} k_{x}^{2} / \mathcal{K}^{2}\right)\left(\bar{\omega}_{\mathrm{p}}^{2} \omega_{1}^{2} \nu^{2}-\delta^{2} k_{x}^{2}|\bar{E}|^{2}\right)}{2 m \gamma_{\mathrm{s}} \omega_{1} \omega_{\mathrm{s}}\left(\omega_{\mathrm{c}}^{2}-\omega_{0}^{2}\right)\left[\left(\delta^{2} \bar{\omega}_{\mathrm{p}}^{2}-k_{x}^{2}|\bar{E}|^{2}\right)^{2}+\left(\omega_{1} \bar{\omega}_{\mathrm{p}}^{2} \nu\right)^{2}\right]}
$$

and

$$
\chi_{\mathrm{EN}_{\mathrm{i}}}^{(2)}=\frac{-e \varepsilon_{\mathrm{L}} A k_{x} \omega_{\mathrm{p}}^{2} \omega_{0}\left(1+L^{2} k_{x}^{2} / \mathcal{K}^{2}\right)\left(\omega_{\mathrm{s}} \omega_{1} \nu^{2}-k_{x}^{2}|\bar{E}|^{2}\right)}{2 m \gamma_{\mathrm{s}} \omega_{1} \omega_{\mathrm{s}}\left(\omega_{\mathrm{c}}^{2}-\omega_{0}^{2}\right)\left[\left(\delta^{2} \bar{\omega}_{\mathrm{p}}^{2}-k_{x}^{2}|\bar{E}|^{2}\right)^{2}+\left(\omega_{1} \bar{\omega}_{\mathrm{p}}^{2} \nu\right)^{2}\right]}
$$

. where $A=\mathcal{K}^{2} k_{x}^{2} v_{x}^{2}$.

For obtaining parametric amplification it is necessary to determine the threshold value of the pump amplitude for the onset of the parametric process. For this setting $\chi_{\mathrm{EN}_{\mathrm{i}}}=0$. The necessary conditions for magnetoactive and isotropic crystals yields:

(i) In the presence of a magnetostatic field, i.e. $\omega_{\mathrm{c}} \neq 0$

$$
\left[\left(E_{0_{\mathrm{ih}}}\right)\right]_{\mathrm{B}_{0} \neq 0}=\frac{m \nu}{e k_{x}}\left(\omega_{1} \omega_{\mathrm{s}}\right)^{1 / 2}\left[1-\left(\omega_{\mathrm{c}} / \omega_{0}\right)^{2}\right] .
$$

(ii) In the absence of a magnetostatic field, i.e. $\omega_{c}=0$

$$
\left[\left(E_{0_{t h}}\right)\right]_{\mathrm{B}_{0} \neq 0}=\frac{m \nu}{e k_{x}}\left(\omega_{1} \omega_{\mathrm{s}}\right)^{1 / 2} .
$$


A comparison of the threshold fields in the presence and absence of the magnetic field $B_{0}$, yields:

$$
\frac{\left[\left(E_{0_{t h}}\right)\right]_{B_{0} \neq 0}}{\left[\left(E_{0_{t h}}\right)\right]_{B_{0}=0}}=\left[1-\left(\omega_{c} / \omega_{0}\right)^{2}\right]
$$

From the above equations one may infer that the application of a magnetostatic field considerably reduces the required value of the threshold pump field. It may also be inferred that the expressions for the threshold fields are independent of the material parameters viz. piezoelectric and/or deformation potential coupling coefficients. Thus it will remain the same for any material belonging to NCS group.

The parametric growth (i.e. $\chi_{\mathbf{E N}_{\mathbf{i}}}$ negative) can be achieved in the two different regions under the following conditions:

$$
\begin{aligned}
& \text { (i) if } k_{x}^{2}|\bar{E}|^{2}>\omega_{1} \omega_{\mathrm{s}} \nu^{2}, \omega_{\mathrm{c}}^{2}<\omega_{0}^{2} \text {; } \\
& \text { (ii) if } k_{x}^{2}|\bar{E}|^{2}<\omega_{1} \omega_{\mathrm{s}} \nu^{2}, \omega_{\mathrm{c}}^{2}>\omega_{0}^{2} .
\end{aligned}
$$

It can be seen that under condition (19b) the expression for the growth becomes nearly independent of the pump field amplitude and thus it is of no practical interest.

Now we shall discuss the different aspects of $\chi_{\mathrm{EN}}$ for different situations of practical interest.

(1) Piezoelectric couplings $\left(\beta \neq 0, C_{\mathrm{d}}=0\right)$ :

For this case, using Eqs. (17) and (19b) one obtains the growth rate as:

$$
g_{\mathrm{p}}=\frac{e^{3} \varepsilon_{\mathrm{L}} A k_{x}^{3} \omega_{\mathrm{p}}^{2} \omega_{0}^{5} \omega_{1} \nu\left|E_{0}\right|^{2}}{2 m^{3} \gamma_{\mathrm{s}} \omega_{\mathrm{s}} \eta c_{0}\left(\omega_{\mathrm{c}}^{2}-\omega_{0}^{2}\right)^{3}}\left[\left(\delta^{2} \bar{\omega}_{\mathrm{p}}^{2}-k_{x}^{2}\left|\bar{E}_{0}\right|^{2}\right)^{2}+\left(\bar{\omega}_{\mathrm{p}}^{2} \omega_{1} \nu\right)^{2}\right]^{-1}
$$

The suffix $\mathrm{p}$ is used for piezoelectric coupling.

(2) Deformation potential coupling $\left(\beta=0, C_{\mathrm{d}} \neq 0\right)$ :

For this case using Eqs. (17) and (19a) one obtains as:

$$
g_{\mathrm{d}}=\frac{e^{3} \varepsilon_{\mathrm{L}} v_{\mathrm{s}}^{2} k_{x}^{7} \omega_{\mathrm{p}}^{2} \omega_{0}^{5} \omega_{1} L^{2} \nu\left|E_{0}\right|^{2}}{2 m^{3} \gamma_{\mathrm{s}} \omega_{\mathrm{s}} \eta c_{0}\left(\omega_{c}^{2}-\omega_{0}^{2}\right)^{3}}\left[\left(\delta^{2} \bar{\omega}_{p}^{2}-k_{x}^{2}\left|\bar{E}_{0}\right|^{2}\right)^{2}+\left(\bar{\omega}_{\mathrm{p}}^{2} \omega_{1} \nu\right)^{2}\right]^{-1} .
$$

The suffix $d$ is used for deformation potential coupling.

(3) Both piezoelectric and deformation potential couplings $\left(\beta \neq 0, C_{\mathrm{d}} \neq 0\right)$ :

In this case using the same pair of equations as above, the gain constant becomes:

$$
g_{\mathrm{b}}=\frac{e^{3} \varepsilon_{\mathrm{L}} A k_{x}^{3} \omega_{\mathrm{p}}^{2} \omega_{0}^{5} \omega_{1} \nu\left|E_{0}\right|^{2}\left[1+L^{2} k_{x}^{2} / \mathcal{K}^{2}\right]}{2 m^{3} \gamma_{\mathrm{s}} \omega_{\mathrm{s}} \eta c_{0}\left(\omega_{\mathrm{c}}^{2}-\omega_{0}^{2}\right)^{3}}\left[\left(\delta^{2} \bar{\omega}_{\mathrm{p}}^{2}-k_{x}^{2}\left|\bar{E}_{0}\right|^{2}\right)^{2}+\left(\bar{\omega}_{\mathrm{p}}^{2} \omega_{1} \nu\right)^{2}\right]^{-1} .
$$

The suffix $\mathrm{b}$ stands for both the couplings. 


\section{Results and discussions}

We now proceed to the numerical analysis of the parametric gain in a NCS semiconducting crystal, viz. $n$-InSb at $77 \mathrm{~K}$ irradiated by pulsed $10.6 \mu \mathrm{m} \mathrm{CO}_{2}$ lasers. The material parameters for this crystal are taken as: $m=0.0150 m_{0}$, $\varepsilon_{1}=15.8, v_{\mathrm{s}}=4 \times 10^{3} \mathrm{~m} \mathrm{~s}^{-1}, \beta=0.054 \mathrm{C} \mathrm{m}^{-2}, C_{\mathrm{d}}=4.5 \mathrm{eV}, \rho=5.8 \times 10^{3} \mathrm{~kg} \mathrm{~m}^{-3}$, $\omega_{\mathrm{s}}=2 \times 10^{11} \mathrm{~s}^{-1}, n_{0}=2 \times 10^{24} \mathrm{~m}^{-3}, \nu=4 \times 10^{11} \mathrm{~s}^{-1}$ and $\omega_{0}=1.78 \times 10^{14} \mathrm{~s}^{-1}$.

Using the above parameters, we have studied the effect of wave number $k$, magnetic field (in terms of $\omega_{c}$ ) and the scattering angle $(\theta)$ on the threshold electric field. Figure 1 shows that the threshold electric field $\left(E_{0_{\mathrm{th}}}\right)$ decreases with the increase in the wave number $k$. It may be inferred from Fig. 2 that $E_{0_{\text {th }}}$ remains constant up to $\omega_{c} \approx 2 \times 10^{13} \mathrm{~s}^{-1}$; if one applies magnetic field for which $\omega_{\mathrm{c}}>2 \times 10^{13} \mathrm{~s}^{-1}$, the $E_{0_{\mathrm{th}}}$ starts decreasing with increament in $\omega_{\mathrm{c}}$. Figure 3 depicts the dependence of $E_{0_{\text {th }}}$ on scattering angle $\theta$. It is observed that for a constant magnetic field, $E_{0_{\mathrm{th}}}$ increases gradually with increasing value of $\theta$.

Considering the pump intensities well above the threshold, the gain constants for all the three types of couplings (i.e. piezoelectric $g_{\mathrm{p}}$, deformation $g_{\mathrm{d}}$ and both $g_{\mathrm{b}}$ ) can be estimated. All the three gain constants increase linearly with the laser pump amplitude $E_{0}\left(>E_{0_{\text {th }}}\right)$ (Fig. 4).

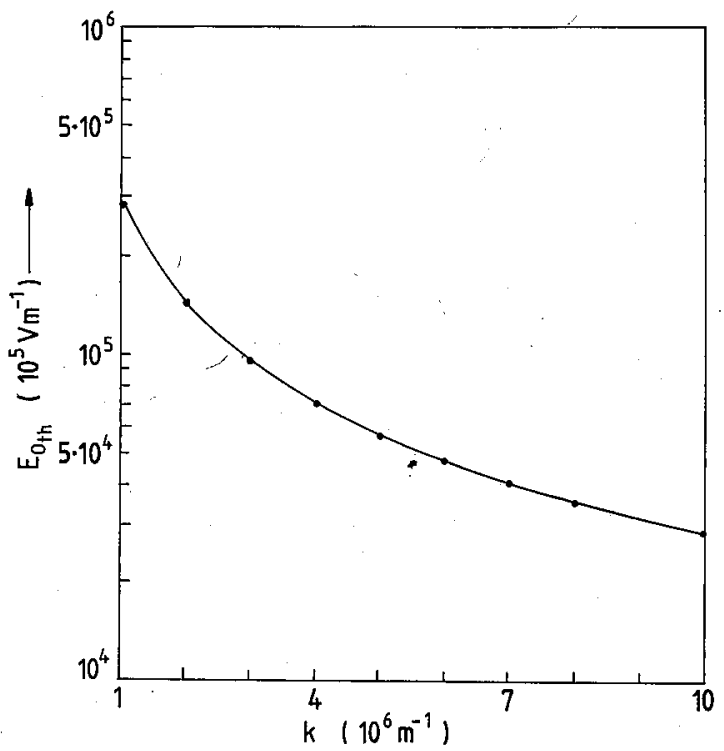

Fig. 1. Variation of threshold field $E_{0_{\text {th }}}$ with wave number $k$ at $\theta=45^{\circ}$ and $\omega_{c}=$ $10^{19} \mathrm{~s}^{-1}$.

It is also found that the values of $g_{\mathrm{b}}$ and $g_{\mathrm{p}}$ are identical and nearly $10^{4}$ order higher than the values of $g_{\mathrm{d}}$ for $k=2 \times 10^{6} \mathrm{~m}^{-1}, \omega_{\mathrm{c}} \approx 2 \times 10^{12} \mathrm{~s}^{-1}$ and 


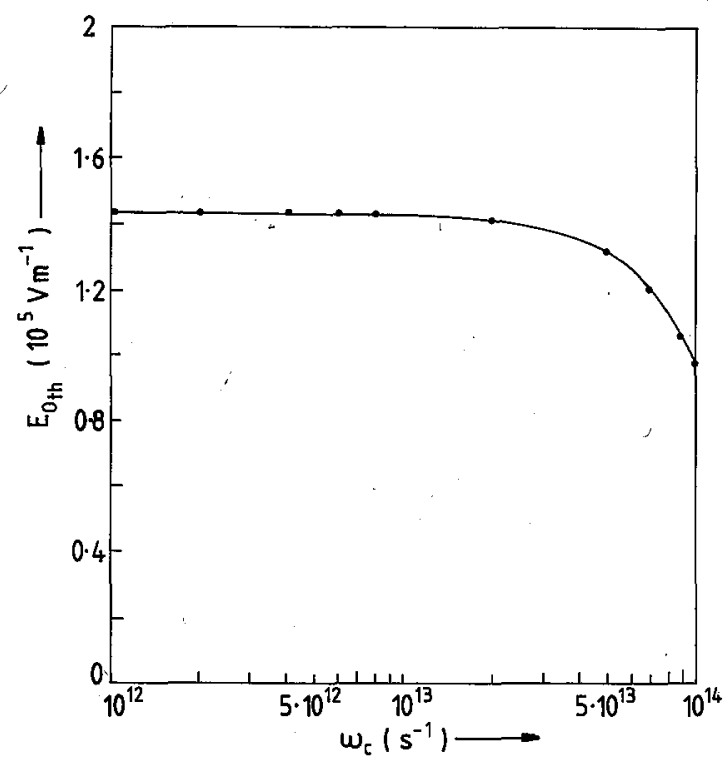

Fig. 2. Variation of threshold field $E_{0_{t h}}$ with magnetic field as measured by $\omega_{c}$ at $\theta=45^{\circ}$ and $k=2 \times 10^{6} \mathrm{~m}^{-1}$.

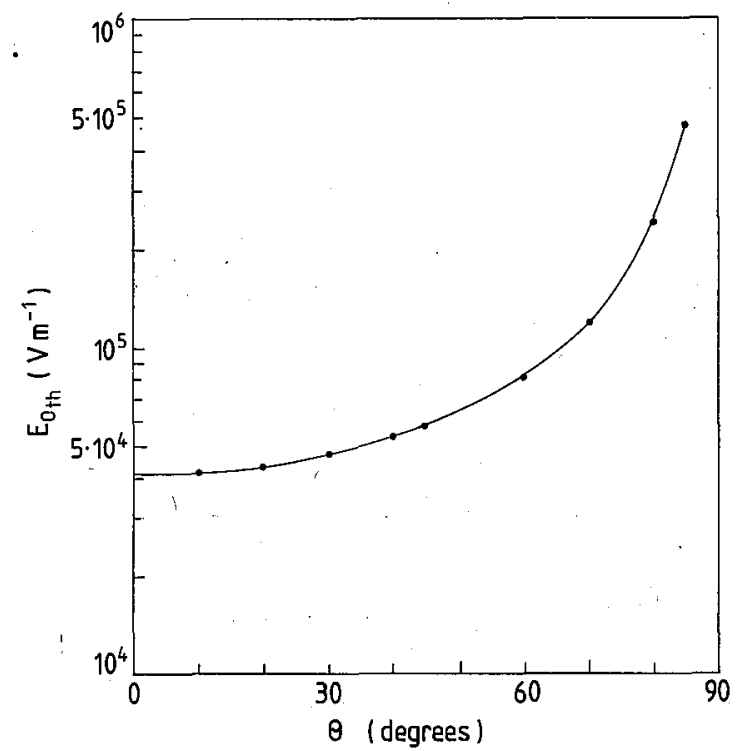

Fig. 3. Variation of threshold field $E_{0_{\mathrm{th}}}$ with angle $\theta$ at $k=5 \times 10^{6} \mathrm{~m}^{-1}$ and $\omega_{\mathrm{c}}=$ $10^{13} \mathrm{~s}^{-1}$. 


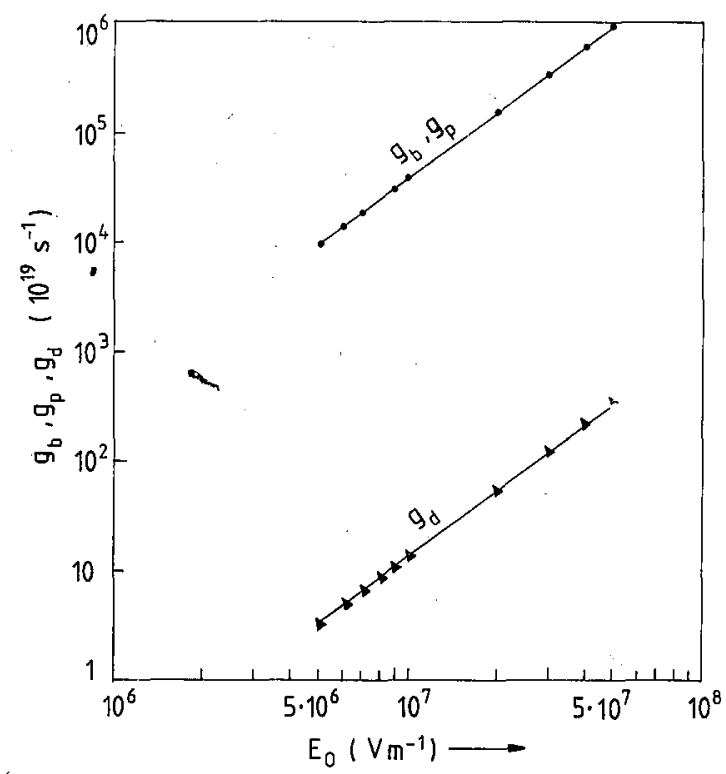

Fig. 4. Variation of parametric gain constants $\left(g_{\mathrm{b}}, g_{\mathrm{p}}\right.$ and $\left.g_{\mathrm{d}}\right)$ with threshold field $E_{0_{\mathrm{th}}}$ at $k=2 \times 10^{6} \mathrm{~m}^{-1}, \omega_{\mathrm{c}}=2 \times 10^{12} \mathrm{~s}^{-1}$ and $\theta=45^{\circ}$.

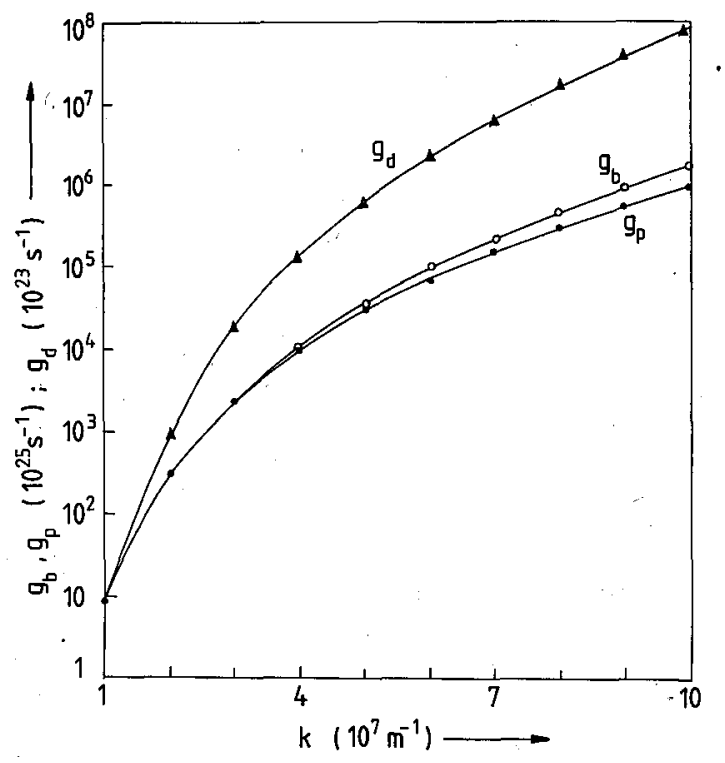

Fig. 5. Variation of parametric gain constants $\left(g_{\mathrm{b}}, g_{\mathrm{p}}\right.$ and $\left.g_{\mathrm{d}}\right)$ with wave number $k$ at $\theta=45^{\circ}, \omega_{c}=10^{12} \mathrm{~s}^{-1}$ and $E_{0}=10^{7} \mathrm{Vm}^{-1}$. 


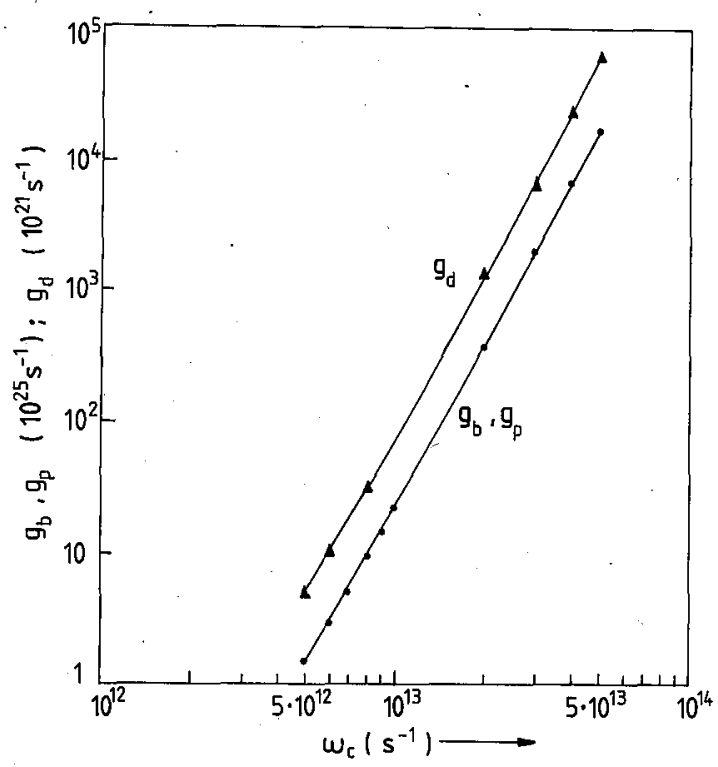

Fig. 6. Variation of parametric gain constants $\left(g_{\mathrm{b}}, g_{\mathrm{p}}\right.$ and $\left.g_{\mathrm{d}}\right)$ with $\omega_{c}$ at $k=2 \times$ $10^{6} \mathrm{~m}^{-1}, \theta=45^{\circ}$ and $E_{0}=10^{7} \mathrm{Vm}^{-1}$.

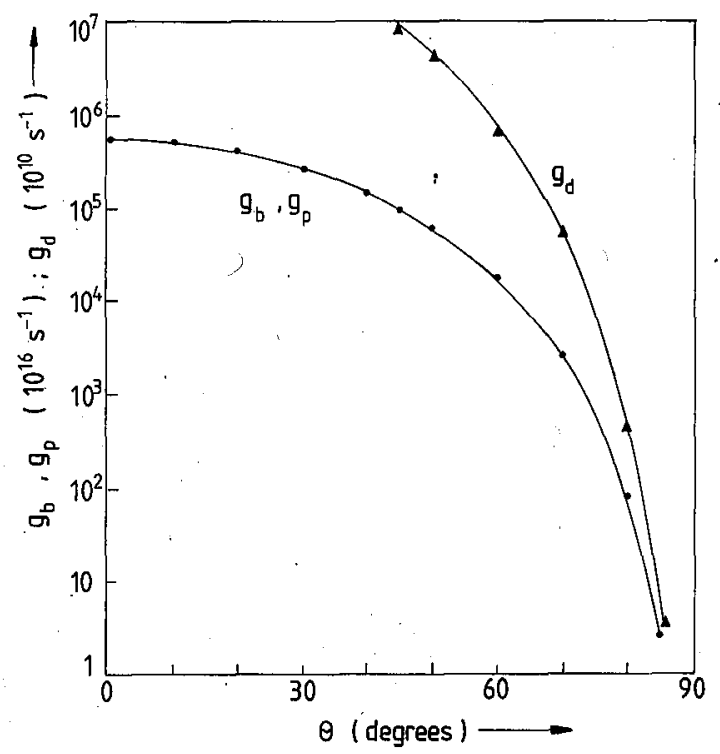

Fig. 7. Variation of parametric gain constants $\left(g_{\mathrm{b}}, g_{\mathrm{p}}\right.$ and $\left.g_{\mathrm{d}}\right)$ with angle $\theta$ at $k=$ $10^{6} \mathrm{~m}^{-1}, \omega_{\mathrm{c}}=10^{12} \mathrm{~s}^{-1}$ and $E_{0}=10^{7} \mathrm{Vm}^{-1}$. 
$\theta=45^{\circ}$. We have plotted the dependence of gain constants on $k$ which varies from $10^{7}$ to $10^{8} \mathrm{~m}^{-1}$ for $E_{0}=10^{7} \mathrm{Vm}^{-1}, \omega_{\mathrm{c}} \approx 2 \times 10^{12} \mathrm{~s}^{-1}$ and $\theta=45^{\circ}$ in Fig. 5. In this wavelength region, i.e. $10^{7}>k>10^{8} \mathrm{~m}^{-1}$, we found that all the three gain constants increase parabolically with $k$. It is also found that $g_{\mathrm{b}}$ and $g_{\mathrm{p}}$ are equal up to $k=3 \times 10^{7} \mathrm{~m}^{-1}$ and for $k>3 \times 10^{7} \mathrm{~m}^{-1}, g_{\mathrm{b}}$ becomes higher than $g_{\mathrm{p}}$. Thus it may be inferred from this figure that the deformation potential coupling becomes comparable to piezoelectric interactions in the wavelength region where $k>3 \times 10^{7} \mathrm{~m}^{-1}$. Below this wa velength region contribution of deformation potential is negligible compared to the contribution of piezoelectricity and thus in this region $g_{\mathrm{p}}$ and $g_{\mathrm{b}}$ are identical. The magnetic field (in terms of $\omega_{\mathrm{c}}$ ) increases all the gain constants (Fig. 6). But we cannot increase $\omega_{\mathrm{c}}$ indefinitely because of the restriction $\omega_{c}^{2}<\omega_{0}^{2}$ (Eq. (19a)). Figure 7 shows the variation of gain constants with the scattering angle $\theta$. It can be inferred from Fig. 7 that the gain constants decrease monotonically with scattering angle, however there is a more rapid decrease for $\theta>30^{\circ}$ than for $\theta<30^{\circ}$.

From the above discussion, it is clear that the required range of threshold electric field $E_{0_{\mathrm{th}}}=2.4 \times 10^{4} \mathrm{~V} \mathrm{~m}^{-1}$ for $k=10^{7} \mathrm{~m}^{-1}$ is corresponding to excitation intensity $4.36 \times 10^{2} \mathrm{~W} \mathrm{~cm}^{-2}$. Such pump intensities are easily obtained by using frequency doubled $\mathrm{CW} 10.6 \mu \mathrm{m} \mathrm{CO}_{2}$ lasers. The most fascinating results obtained in the study reported above are as follows:

(1) The applied magnetic field increases the values of gain constants, while it reduces the required pump intensities.

(2) The gains are maximum whereas the required threshold intensity is minimum for zero scattering angle, i.e. one gets maximum gain for minimum threshold for backward scattered mode.

(3) It is found that the gains with piezoelectricity $g_{\mathrm{p}}$ and with both couplings $g_{\mathrm{b}}$ are identical up to the wave number $k=3 \times 10^{7} \mathrm{~m}^{-1}$ and beyond this value of $k$, $g_{\mathrm{b}}$ becomes more than $g_{\mathrm{p}}$.

(4) The gain with deformation potential $g_{\mathrm{d}}$ is always found to be less than that of $g_{\mathrm{p}}$ and $g_{\mathrm{b}}$.

Thus one may conclude from the above discussion that the magnetoactive, highly doped semiconductors, having both the coupling mechanisms are the best choice for the fabrication of parametric backward wave amplifiers.

\section{References}

[1] V.I. Vespalov, A.M. Kiseljov, G.A. Pasmanik, Proc. SPIE 478, 286 (1984).

[2] F.A. Hopf, A. Tomita, T. Lipman, Optics Commun. 37; 72 (1981).

[3] A. Piskarsks, A. Stabinis, A. Yankauskas, Sov. Phys. JETP 29, 869 (1986).

[4] Ch. Flytzanis, in: Quantum Electronics, Vol. I, eds. H. Rabin, C.L. Tang, Academic Press, New York 1975 p. 9.

[5] C.Y. Fang, Y.R. Shen, Phys. Rev. Lett. B 12, 2325 (1975). 
[6] F.G. Parsons, E.Y. Chen, R.K. Chang, Phys. Rev. Lett. 27, 1436 (1971).

[7] K.E. Pieponen, J. Phys. C 20, 285 (1987).

[8] K.E. Economou, H.N. Spector, Phys. Rev. B 18, 5578 (1978).

[9] B.I. Cohen, Phys. Fluids 30, 2676 (1987).

[10] S. Ghosh, V.K. Agarwal, Acta Phys. Pol. A63, 587 (1983).

[11] P. Aghamkar, P. Sen, P.K. Sen, Phys. Status Solidi B 145, 343 (1988).

[12] C.N. Lashmore Davies, J. Plasma Phys. 17, 281 (1975).

[13] M.C. Steele, B. Vural, Wave Interactions in Solid State Plasmas, Mc Graw Hill, New York 1969, p. 136.

[14] S. Guha, P.K. Sen, S. Ghosh, Phys. Status Solidi A 52, 407 (1979). 